\title{
Damping Behavior of Synthetic Graphite Beams
}

\author{
Luiz Cláudio Pardini ${ }^{\mathrm{a} *}$, Flaminio Levy Neto ${ }^{\mathrm{b}}$, Jorge Luiz de Almeida Fereira ${ }^{\mathrm{b}}$ \\ ${ }^{a}$ CTA/IAE/AMR, 12225-904 São José dos Campos - SP, Brazil \\ ${ }^{\mathrm{b}}$ UnB/FT/ENM, 70910-900 Brasilia - DF, Brazil
}

Received: July 7, 2005; Revised: October 3, 2005

\begin{abstract}
The main objective of this work was to obtain the damping factor $(\xi)$ as well as the elasticity modulus (E) of two kinds of synthetic graphite (HLM and ATJ), using the modal analysis technique. Prismatic beams of square section $(\sim 11 \times 11 \mathrm{~mm})$ and length over thickness ratio $(\mathrm{L} / \mathrm{t})$ of about 22.7 were tested in the free - free boundary condition. The first four modes of vibration were taken into account in the non-destructive evaluation of the materials. In addition, numerical simulations were also carried out in this investigation. The agreement between the theoretical and the experimental results was quite good. The average values of $\mathrm{E}$ and $\xi$ for the HLM graphite were $20 \%$ and $90 \%$ higher, respectively, than those presented by the ATJ graphite, indicating that the HLM graphite has, proportionally, more damping mechanisms than the ATJ graphite.
\end{abstract}

Keywords: synthetic graphite, modal analysis, vibration damping

\section{Introduction}

Graphite is a material that has shown a history of continually widening technological applications. Originally developed for use as electrodes, it now has many uses which include: neutron moderation in nuclear power reactors; rocket nozzle throats for space vehicles; brushes of electrical machines; crucibles for high temperature use; self lubricating bearings; electrodes for plasma arc carbon nanotubes synthesis, and many other applications.

In its natural form, graphite is an allotropic form of the element carbon that shows a well developed layered structure, stacked parallel to each other in the sequence $\mathrm{ABAB} . .$. , in which the atoms are hexagonally arranged forming the so called basal planes ${ }^{5,9}$, as shown schematically in Figure 1. Due to the fact that the chemical bonds within the layers are covalent, but, on the other hand, those between the layers are weak forces of van der Waals, natural graphite is anisotropic. In particular, it presents higher strength and stiffness in basal planes and, in comparison, poor mechanical behavior perpendicularly to the basal planes. Synthetic graphites, which are particulate composites, present a smaller degree of discrepancy among the mechanical properties, and so, macroscopically, they exhibit a mechanical behavior closer to the isotropic.

Synthetic graphites can be obtained from a mixture of coke and pitch binder, with small amounts of natural graphite, which is subjected to a series of thermal and mechanical treatments that ends with a graphitization process at a temperature of approximately $3000{ }^{\circ} \mathrm{C}^{4}$. Despite the fact that the mechanical properties of graphite, at about $20{ }^{\circ} \mathrm{C}$, can be considered only moderate $(10<\mathrm{E}<12 \mathrm{GPa}$, tensile static strength of about $40 \mathrm{MPa}$ ), they can maintain such properties up to temperatures of about $2000{ }^{\circ} \mathrm{C}$, in the absence of an oxidising atmosphere $^{6,7}$. In addition, the density of graphite is very low, typically below $2 \mathrm{~g} / \mathrm{cm}^{3}$, which make this material suitable for aerospace applications.

Graphites can be classified, according to the raw materials, as coarse, medium or fine grain, and, as far as their processing technique are concerned, as extruded or molded grades. In addition, depending on the conditions of the heat treatments during processing, there are different categories of synthetic graphite. In the present study, two kinds of commercial synthetic graphite will be investigated: (i) extruded graphite with coarse grains, designated as HLM; and (ii) a graphite which was subjected to isotropic compression during the manufacture process, presenting fine grains and designated as ATJ.

Despite the fact that details of the static behavior of graphites is available in some publications ${ }^{5,2,9}$, few works have addressed the dynamical behavior of these materials in the literature so far, probably because their primarily use was mainly for thermal management in electric arc furnaces. In this investigation, the elasticity modulus (E) and the damping factor $(\xi)$ of two kinds of synthetic graphites (HLM and ATJ) will be evaluated, using the technique of modal analysis. Such parameters (E and $\xi$ ), among others, are important for the design of nozzle throats of rocket engines which, in particular, are continuously subjected to mechanical vibrations and high temperatures. In this context, the main objective of this research was to carry out non-destructive experiments and numerical simulations, starting from scratch, in order to obtain the damping factor, as well as the elasticity modulus, of prismatic beams of synthetic graphite.

\section{Materials and Methods}

The specimens used in this study consisted of prismatic beams of HLM 85, from Sigri-Great Lakes Co. and ATJ synthetic graphite, from UCAR Co The Figure 2 shows typical optical micrographs of polished surfaces of the HLM graphite (Figure 1a) and ATJ graphite (Figure $2 \mathrm{~b}$ ). The processing conditions and the raw materials are key issues in determining characteristic features of materials and so it happens to graphites. Graphites are composed mainly by coke particles and pyrolised pitch. The coke particles accounts for almost $80 \%$ in weight. So, the size and type of the coke particles and their size distribution are important during the molding process. The molding process can be either by compression molding or by extrusion, which in most of the cases tends to align the coke particles. Moreover, the higher is the level of heat treatment temperature the better are thermal and mechanical properties, which is a result of basal plane alignments.

The HLM graphite is formed by a binder phase and elongated needled-coke grains. As a consequence HLM graphite is anisotropic 
in nature. Binder phase regions and filler particles for graphites are more clearly seen under polarized microscopy (reflection interference colors), which is not shown, and they exhibit a broad variation in size

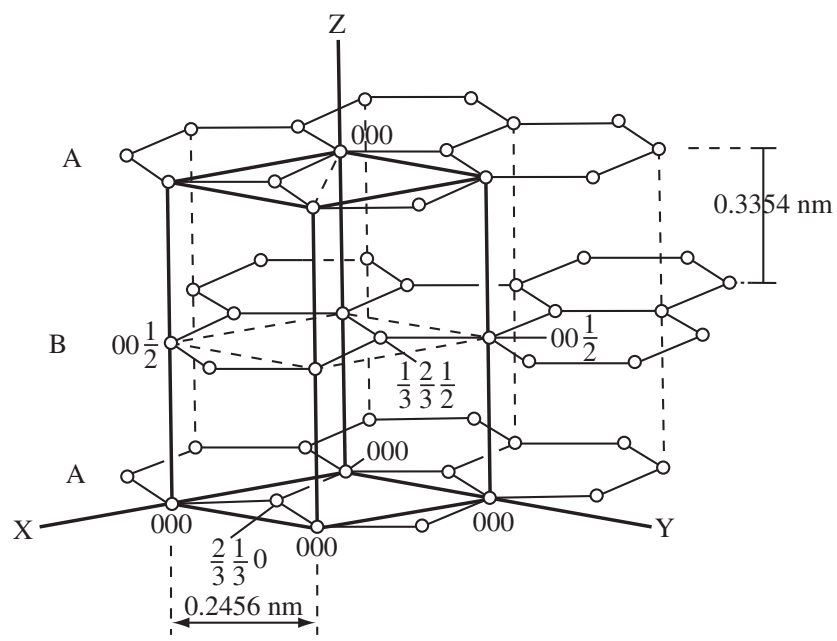

Figure 1. Representation of an ideal lattice of a graphite.

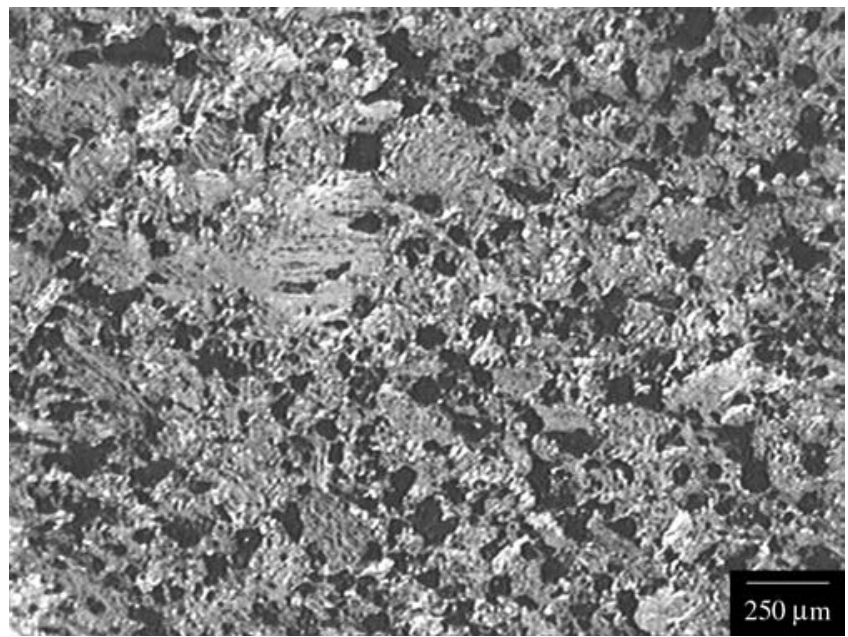

(a)

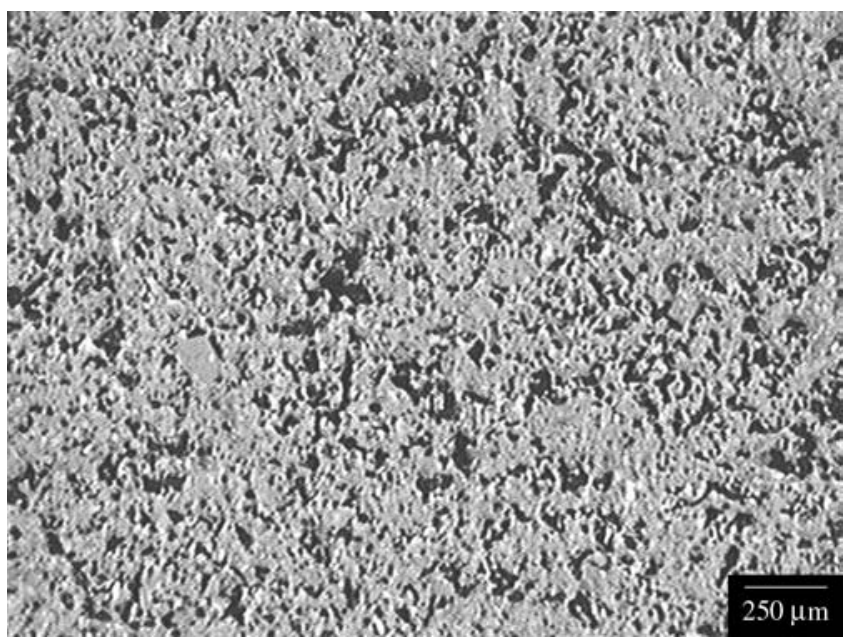

(b)

Figure 2. a) Optical microscopy for the HLM; and b) ATJ graphite. Dark areas represents pores. and shape. A typical size of commom basal plane orientation of these anisotropic components is $\sim 125 \mu \mathrm{m}$, which is in the range of $1-500 \mu \mathrm{m}$ as described by Forest and Marsh ${ }^{11}$. In fact, the initial medium grain size for the HLM graphite from manufacturer's data sheet is $0.8382 \mathrm{~mm}$. A broad range of pores and microcracks, represented by dark areas in Figure 2a, can be found in the microstructure HLM graphite. Mercury intrusion porosimetry shows that a $12 \%$ open pore volume is present in the HLM graphite. The IUPAC boundary for mesopores and macropores is at pore radius $\sim 25 \mu \mathrm{m}$. The pore distribution size is broad, ranging from 1-2 $\mu \mathrm{m}$ to $250 \mu \mathrm{m}$.as shown in Figure 2a.

ATJ graphite (Figure 2b) has a quite different microstructure in relation to the HLM graphite. ATJ graphite exhibits a more homogeneous structure and pores are smaller, most of then are elongated, than the HLM graphite. The filler particles in the ATJ graphite are hardly distinguished at the level of magnification used for observation. In general, the lower the size of the coke particles from which graphite is composed the higher is their isotropy, which is translated to mechanical and thermal properties, regardless the molding process used. Due to this fact, ATJ graphites can be suitable for use in gas cooled nuclear reactors. Thus, it appears that the coke particles used in the ATJ graphite manufacture are significantly smaller than the ones used for the HLM manufacture. Mercury intrusion porosimetry shows that a $8 \%$ open pore volume is present in the ATJ graphite. The majority of the pores are mainly mesopores which are in the range of 1 to $10 \mu \mathrm{m}$.

Graphite samples were machined to the nominal dimensions $11 \mathrm{x}$ $11 \times 250 \mathrm{~mm}$, and tested in the vertical position (direction Z), with a the free-free boundary condition, using two elastic strings at each extremity of the test specimens, as shown in Figure 3. This configuration in the tests was adopted after an extensive series of preliminary experiments was carried out.

In order to evaluate the dynamic parameters of the beams, the experimental modal analysis was carried out exciting the structure with an instrumented hammer (PCB Piezotronics), according to the set up represented in Figure 3. The hammer had a sensibility of $0.18 \mathrm{mV} / \mathrm{N}$ and its plastic tip was chosen in order to generate a well-defined spectrum in the band varying from 0 to $5 \mathrm{kHz}$, considered in this study. The average mass of the tested beams was about 55.2 grams, and the chosen accelerometer (Piezotronics 353B16, sensibility of $10.31 \mathrm{mV} / \mathrm{g}$ ) had a mass of 1.5 grams. Taking into account the relative dimensions of the accelerometer and the graphite beams, the number of drive points adopted in the experiments for the dynamical measurements was eleven. Briefly, for each one of the equally spaced 11 drive points, successively, the following steps were executed during the course of the experiments:

1. Fixation of the accelerometer in the first drive point, with its longitudinal axis perpendicularly to the surface of the beam, using wax;

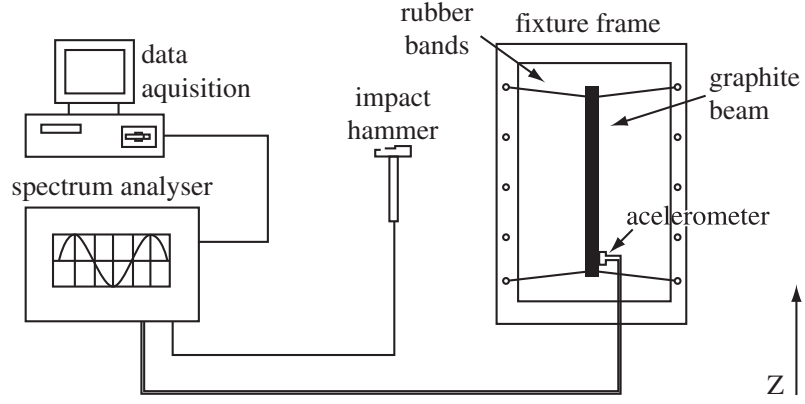

Figure 3. General view of the test assembly. 
2. Excitation of the first test point with the instrumented hammer, verifying, after the impact, by inspection of the temporal response signal, if multiple impacts did not occur;

3. Repeat step 2 five times;

4. Processing of the Frequency Response Function (FRF) and the coherence between excitation signal of the test point (i.e. the force from the hammer) and the acceleration from the drive point, based on the average of the five impacts (step 3); and

5. Repetition of steps 2, 3 and 4, for the remaining 10 test points.

The commercial software STARmodal from Structural Measurements Systems Inc was used to execute the processing of the data obtained from the tests. The dynamical analyser used in the experiments was the Hewlett Packard (HP) model 35665A and the first four modes of the beams were considered in this study. For the initial validation of the experiments, the test results were compared with classical solutions for the dynamical behavior of beams found in the literature ${ }^{8}$. In addition, the results were also compared with numerical simulations of the problem using the finite element (FE) code ANSYS/ED, adopting an eight nodes plane stress element of constant thickness in the FE mesh. In such simulations it was possible to obtain the natural frequencies and the modes of vibration. These two spectral parameters were analysed and also compared with those obtained experimentally.

Based on each natural frequency obtained experimentally, the elasticity modulus (E) of a prismatic beam can be estimated by Equation $1^{1,8}$. In the present study, the first four modes of vibration, associated with their natural frequencies, were taken into account in the analisys.

$$
E=\frac{\rho \cdot A \cdot \omega_{i}^{2} \cdot l^{4}}{I \cdot(\beta \cdot l)_{i}^{4}}
$$

where: $\rho$ is the specific mass; $A$ is the cross section area; $\omega_{i}$ is the natural frequency associated to the order of the vibration mode; $l$ is the length of the beam; $I$ is the moment of inertia of the cross section area; and $(\beta \cdot l)$ is a constant associated to the order of the vibration mode and the boundary conditions. For the free-free boundary condition, the $(\beta \cdot l)$ constants are equal to 3.9266 in the $1^{\text {st }}$ mode, 7.0685 in the $2^{\text {nd }}$ mode, 10.2101 in the $3^{\text {rd }}$ mode, and 13.3517 in the $4^{\text {th }}$ mode, respectively.

The typical characteristics of the beams used in the experimental study are show in Table 1. The density of the HLM and the ATJ graphite beams was estimated using their dimensions and the weights, while the range of the dynamic modulus (E) was obtained in the literature ${ }^{6}$.

In order to evaluate the quality of the experimental results, the degree of correlation between the experimental modal shapes and those obtained numerically were compared using the Modal Assurance Criterion (MAC), which is also known as Mode Shape Correlation Coefficient (MSCC) ${ }^{3,10}$. This criterion generates a parameter that indicates the degree of correlation between the mode $\mathrm{j}$ of the first test and mode $\mathrm{k}$ of the second test; varying their values from 0 to 1 , with 0 for no correlation and 1 for total correlation. Let $\phi_{A}$ and $\phi_{B}$ be the first and the second modes of vibration written as $\phi_{A}[n \times m]$ and $\phi_{B}[n \times m]$,

Table 1. Characteristics and Elasticity Modulus of the synthetic graphite beams.

\begin{tabular}{lcccc}
\hline Graphite & $\begin{array}{c}\text { Length } \\
(\mathrm{mm})\end{array}$ & $\begin{array}{c}\text { Height } \\
(\mathrm{mm})\end{array}$ & $\mathrm{E}(\mathrm{GPa})$ & $\begin{array}{c}\text { Density } \\
\left(\mathrm{Kg} / \mathrm{m}^{3}\right)\end{array}$ \\
\hline HLM & 250 & 11.10 & $10<\mathrm{E}<12$ & 1731.43 \\
ATJ & 250 & 11.45 & $10<\mathrm{E}<12$ & 1740.83 \\
\hline
\end{tabular}

where $\mathrm{m}$ is the number of modes and $\mathrm{n}$ is the number of nodal points analysed. The MAC is then defined for $\mathrm{j}$ and $\mathrm{k}$ as follows:

$$
\operatorname{MAC}(j, k)=\left(\Sigma_{i i}^{n} \phi_{A j} \cdot{ }_{i} \phi_{B k}\right)^{2} /\left(\Sigma_{i}^{n}\left({ }_{i} \phi_{A j}\right)^{2} \cdot \sum_{i}^{n}\left({ }_{i} \phi_{B k}\right)^{2}\right)
$$

where: ${ }_{i} \phi_{A j}$ is the coordinate $i$ of the column $j$ of $\phi_{A}$ and $\phi_{B k}$ the coordinate $i$ of column $k$ of $\phi_{B}$.

As one can observe in Equation 2 the MAC is based on the scalar product between two unitary vectors. So, if such measure is equal to zero it can be assumed that the vectors are orthogonal. If the MAC between two modal shapes is equal to 1.0 , then such vectors are perfectly correlated. In practice, any value between 0.9 and 1.0 is considered to be a good correlation (HP Application Note, 243-3, 1986). If the value of the coefficient is smaller than 0.9 , then there will be a degree of inconsistency which will greater the smaller is the value of the coefficient. So, the MAC is important to evaluate the correlation between the numerical and experimental modal shapes.

In addition to the MAC, the Modal Scale Factor (MSF) (HP Application Note 243-3, 1986, Ewins $)^{3}$ was also used in order to evaluate the agreement between the estimated experimental and numerical natural modes of vibration. Put in a simple way, the MSF represents the inclination of the best-fitted straight line that represents the points of the graphic. The quantification of this parameter can be obtained from Equation 3:

$$
\operatorname{MSF}(x, p)=\frac{\sum_{i=1}^{N}\left(\phi_{x}\right)_{i}\left(\phi_{p}\right)_{i}^{*}}{\sum_{i=1}^{N}\left(\phi_{p}\right)_{i}\left(\phi_{p}\right)_{i}^{*}}
$$

\section{Results and Discussions}

The experimental and numerical (FE) natural frequencies, as well as the experimental damping factor of the HLM and ATJ graphite beams, for the first four vibration modes, are presented in Tables 2 and 3, respectively. The damping factors presented in these Tables suggests that the damping characteristics of the HLM graphite, in the range of frequencies covered in the experiments (from about 440 to $4300 \mathrm{~Hz}$ ), are more attractive than those presented by the ATJ graphite. The average damping factor of the HLM graphite $(0.724$ $\pm 0.135 \%$ ) was about $119 \%$ higher than the average damping factor of the ATJ graphite $(0.331 \pm 0.061 \%)$. The HLM graphite (average E equal to $11.63 \mathrm{GPa}$ ) was also stiffer than the ATJ (average E equal to $9.71 \mathrm{GPa}$ ) and, quantitatively, in the experiments carried out so far, presented a dynamic elasticity modulus, which, in average, was $20 \%$ higher.

Comparing the numerical and the experimental results of the first four natural frequencies of the graphite beams, presented in Tables 2 and 3 , it can be verified that the maximum difference observed between them is close to $9 \%$, while the average difference is about $6 \%$. This indicated that the finite element simulation model was fairly accurate in the range of frequencies investigated in this study, which included the fist four modes of vibration of the graphite beams. Based

Table 2. Dynamical characteristics estimated for the HLM graphite beam.

\begin{tabular}{crrrc}
$\begin{array}{c}\text { Vibration } \\
\text { modes }\end{array}$ & \multicolumn{2}{c}{$\omega_{\mathrm{n}}(\mathrm{Hz})$} & $\xi-(\%)$ & $\begin{array}{c}\mathrm{E}(\mathrm{GPa}) \text { Based on } \\
\text { Equation 1 }\end{array}$ \\
& \multicolumn{1}{c}{ Exp. } & $\mathrm{FE}$ & & \\
\hline $1^{\circ}$ mode & 512.44 & 475.52 & 0.62274 & 12.50 \\
$2^{\circ}$ mode & 1371.00 & 1296.00 & 0.81372 & 11.78 \\
$3^{\circ}$ mode & 2745.45 & 2503.00 & 0.56600 & 10.99 \\
$4^{\circ}$ mode & 4318.18 & 4059.00 & 0.89503 & 11.26 \\
\hline
\end{tabular}


on Equation 1 and the frequencies presented on Tables 2 and 3 the mean values of the elasticity modulus of the graphite HLM and ATJ were estimated, respectively, as about 11.6 and 9.7 GPa.

The MAC and MSF values involving the numerical and experimental modal vectors for the HLM and the ATJ beams are presented in Tables 4 and 5, respectively. According to the data presented in these Tables, there was an excellent correlation between the vectors, since the MAC and MSF values in all the analyzed modes are very close to 1.0. It was also observed that the largest deviations from the best straight line matching the experimental points and the ideal theoretical straight line obtained numerically occur for the modes 2 and 4 . However, the good correlations obtained so far were the result of an extensive series of experiments and simulations.

Initially, the measurements involving all the eleven nodes of the tested beams, for all the four vibration modes taken into account, were included in the modal analysis, and the estimations of the elasticity modulus of the graphites HLM and ATJ were not in good agreement with those obtained from the literature. It was observed that, for the nodes along the direction $\mathrm{Z}$ shown in Figure 4, each vibration mode presents points of relatively large amplitudes, and points of stagnation (in which the amplitudes are negligible, relatively). Since the points of higher amplitudes tend to present a more favorable signal to noise ratio, increasing the precision of the measurements, significantly; and for the points of much lower amplitude the opposite trend takes place, the points of stagnation (e.g. points 1, 6 and 11, in Figure 4) were eliminated from the analysis. As a result, only when the points of so called "good displacement" (e.g. points of higher amplitudes: 2, 5, 7 and 10, in Figure 3) were considered in the modal analysis, the correlations involving the comparison of the experimental elasticity modulus with those from the literature improved significantly and presented good agreement with those obtained from the literature.

Table 3. Dynamical characteristics estimated for the ATJ graphite beam.

\begin{tabular}{crccc}
\hline $\begin{array}{c}\text { Vibration } \\
\text { modes }\end{array}$ & \multicolumn{2}{c}{$\omega_{\mathrm{n}}(\mathrm{Hz})$} & $\xi-(\%)$ & $\begin{array}{c}\text { E }(\mathrm{GPa}) \text { Based } \\
\text { on Equation 1 }\end{array}$ \\
& \multicolumn{1}{c}{ Exp. } & \multicolumn{1}{c}{$\mathrm{FE}$} & & \\
\hline $1^{\mathrm{o}}$ mode & 463.93 & 441.74 & 0.45056 & 10.25 \\
$2^{\text {o }}$ mode & 1229.09 & 1203.60 & 0.34874 & 9.47 \\
$3^{\text {o mode }}$ & 2464.55 & 2321.8 & 0.29796 & 9.91 \\
$4^{\mathrm{o}}$ mode & 3927.27 & 3761.00 & 0.42598 & 9.21 \\
\hline
\end{tabular}

Table 4. MAC and MSF values between the numerical and experimental vectors for HLM graphite beam.

\begin{tabular}{ccc}
\hline Mode & MAC & MSF \\
\hline $1^{\circ}$ & 0.998115 & 0.999057 \\
$2^{\circ}$ & 0.981135 & 0.990523 \\
$3^{\circ}$ & 0.983119 & 0.991523 \\
$4^{\circ}$ & 0.978570 & 0.989227 \\
\hline
\end{tabular}

Table 5. MAC and MSF values between the numerical and experimental vectors for HLM graphite beam.

\begin{tabular}{ccc}
\hline Mode & MAC & MSF \\
\hline $1^{\circ}$ & 0.998120 & 0.999060 \\
$2^{\circ}$ & 0.994070 & 0.997031 \\
$3^{\circ}$ & 0.979822 & 0.989860 \\
$4^{\circ}$ & 0.982981 & 0.991454 \\
\hline
\end{tabular}

In Figure 4, which refers to the fourth vibration mode of the beam, the points 2, 5, 7 and 10 were considered points of "good displacement", whereas the points of negligible amplitute, 1, 6 and 11, on the other hand, were treated as points of stagnation and eliminated from the analysis.

\section{Conclusions}

This work presented the results obtained using an experimental non-destructive technique known as modal analysis, which allows one to obtain the damping factor $(\xi)$, as well as the dynamic modulus (E) of synthetic graphites. The elastic modulus, alternatively, could also be measured with the use of strain gages glued in bars (or beams) subjected to static known forces. However, graphite is a very brittle material and fractures at very low strains. So, it is not an easy task to obtain the elastic modulus of a graphite beam, with good accuracy, from the measurement of its tensile (or bending) strains under the action of a known force, without destroying the test specimen in the first place. Cracks and pores are common features that are found in graphite microstructure, and graphite bars and beams are prone to fail when they are subjected to very low strains. In addition, the modal analysis is cheaper, and allows one to obtain both properties, E and $\xi$, in a single test.

The experimental values of $\mathrm{E}$, for both kinds of graphite, presented good correlation with data available in the literature ${ }^{6,7}$, and the scatter of the results, taking into account all the four vibration modes considered in the analysis, was always less than 5\%. The HLM coarse grain grain graphite presented an average dynamic modulus (E) higher than the one concerned with the fine grain ATJ by a factor of 1.20. In addition, the average damping factor $(\xi)$ of the HLM graphite was about $119 \%$ higher in comparison with the value obtained for the ATJ graphite. The scatter in the values of $\xi$ were in the range from about 16 to $19 \%$. However, since the synthetic graphites are porous particulate composites with presents some degree of residual stresses from the heat treatments which they are subjected ${ }^{5,9}$, such range of magnitudes in the scatter for the damping factors are not a surprise. Finally, as far as the values of E and $\xi$ are concerned, the HLM graphite can be considered slightly superior in relation to the ATJ graphite for thermo-structural applications. Synthetic graphites are anisotropic in nature depending on their molding conditions. So, the apparent superior elastic properties of the HLM graphite beam must be treated with caution. In order to obtain both the longitudinal as well as the transverse properties of these two kinds of graphite, it is necessary to test plates of these materials and to carry out a bidimensional, or 2-D, analysis. However, such study, at this moment, is still beyond the scope of the present 1-D analysis.

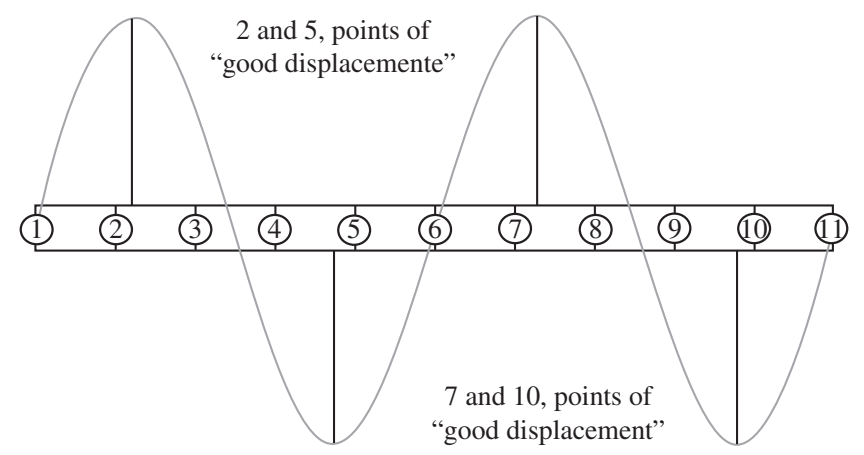

Figure 4. Points of "good displacement" for the fourth mode of vibration of the beam. 


\section{Acknowledgment}

The authors of this work are grateful for the support given by CNPq to the project 520102/98.

\section{References}

1. Adorno TB. Experimental and Numerical Analysis of Graphitic Components. Bachelor of Mechanical Engineering Report; Distrito Federal: Departamento de Engenharia Mecânica, Universidade de Brasilia; 2002.

2. Blackman LCF. Modern Aspects of Carbon and Graphite Technology. London: Academic Press; 1970.

3. Ewins DJ. Modal Testing: Theory and Practice. New York: John Wiley \& Sons; 1984.

4. Friedrich C. et al. The Wold's First $800 \mathrm{~mm}$ Diameter Graphite Electrode for a DC Electric Furnace. MTP International. 2002; 23(2):42-49.

5. Marsh H. Introduction to Carbon Science. London: Buttherworths; 1989.
6. Pardini LC, Manhani LGB. Analysis of Dynamic Mechanical Properties of Synthetic Graphites; 2001 Oct 15-17; I Post-Graduate Meeting of Universidade do Vale do Paraíba, São José dos Campos: São Paulo; 2001.

7. Polidoro HA. Development of Electrical Rods with Resistive Element of Synthetic Graphite or Carbon-Carbon Composites for Simulation Tests of Transients in Nuclear Reactors. [unpublished D.Sc thesis]. São José dos Campos: Instituto Tecnológico de Aeronáutica; 1987.

8. Rao SS. Mechanical Vibrations. New York: Addison-Wesley; 1995.

9. Reynolds WN. Physical Properties of Graphite. London: Elsevier; 1968.

10. Sreenivas A, Fu G, Dillon EW. Signal versus noise in damage detection by experimental modal analysis. Journal of Structural Engineering. 1997; 123(2):237-245.

11. Forest RA, Marsh H. Reflection Interference Colours in Optical. Microscopy. of. Carbon. Carbon. 1977; 15:348-349. 